\title{
Exploratory study of how cognitive multisensory rehabilitation restores parietal operculum connectivity and improves upper limb movements in chronic stroke
}

\section{Ann Van de Winckel ( $\square$ avandewi@umn.edu )}

Division of Physical Therapy, Division of Rehabilitation Science, Department of Rehabilitation Medicine, Medical School, University of Minnesota, USA https://orcid.org/0000-0001-8400-3177

\section{Daniele De Patre}

Centro Studi di Riabilitazione Neurocognitiva - Villa Miari (Study Center for Cognitive Multisensory Rehabilitation), Santorso, Vicenza, Italy

\section{Marco Rigoni}

Centro Studi di Riabilitazione Neurocognitiva - Villa Miari (Study Center for Cognitive Multisensory Rehabilitation), Santorso, Vicenza, Italy

\section{Marc Fiecas}

Division of Biostatistics, School of Public Health, University of Minnesota, USA https://orcid.org/00000002-6472-9410

\section{Timothy J Hendrickson}

University of Minnesota Informatics Institute, Office of the Vice President for Research, University of Minnesota, USA

\section{Mia Larson}

Division of Rehabilitation Science, Department of Rehabilitation Medicine, Medical School, University of Minnesota, USA

\section{Bharathi D Jagadeesan}

Department of Radiology, Medical School, University of Minnesota, USA https://orcid.org/0000-00021143-941X

\section{Byron A Mueller}

Department of Psychiatry, Medical School, University of Minnesota, USA https://orcid.org/0000-00032429-0391

\section{Wendy Elvendahl}

Center of Magnetic Resonance Research (CMRR), University of Minnesota, USA

\section{Christopher Streib}

Department of Neurology, Medical School, University of Minnesota, USA https://orcid.org/0000-00021119-757X

\section{Farha Ikramuddin}


Division of Physical Medicine \& Rehabilitation, Department of Rehabilitation Medicine, Medical School, University of Minnesota, USA https://orcid.org/0000-0003-4507-8074

\section{Kelvin 0 Lim}

Department of Psychiatry, Medical School, University of Minnesota, USA https://orcid.org/0000-00022390-7268

\section{Research Article}

Keywords: stroke, functional brain imaging, paresis, rehabilitation, recovery of function, therapy, upper limb, parietal operculum

Posted Date: September 10th, 2020

DOI: https://doi.org/10.21203/rs.3.rs-75235/v1

License: (c) (i) This work is licensed under a Creative Commons Attribution 4.0 International License. Read Full License 


\section{Abstract}

Cognitive multisensory rehabilitation (CMR) is a promising therapy for upper limb recovery in stroke, but the brain mechanisms are unknown. We previously demonstrated that the parietal operculum (parts OP1/OP4) is activated with CMR exercises. In this exploratory study, we assessed the baseline difference between OP1/OP4 functional connectivity (FC) in the brain at rest in stroke versus healthy adults to then explore whether CMR affects OP1/OP4 connectivity and motor recovery after stroke.

We recruited 8 adults with chronic stroke and left hemiplegia/paresis and 22 healthy adults. Resting-state FC with the OP1/OP4 region-of-interest in the affected hemisphere was analysed before and after 6 weeks of CMR. We evaluated sensorimotor function and activities of daily life pre- and post-CMR, and at 1-year post-CMR.

We found decreased FC between the right OP1/OP4 and 34 areas distributed across all lobes in stroke versus healthy adults. After $\mathrm{CMR}$, only four areas had decreased $\mathrm{FC}$ compared to healthy adults. Participants improved on motor function (MESUPES, $p=0.03$; Fugl-Meyer, $p=0.006$ ), with those improvements maintained at 1-year follow-up. Stereognosis and Frenchay test (both $p=0.03$ ) improved from pre-CMR to 1-year follow-up, suggesting enhancing sensorimotor recovery post-stroke by CMR. Our results from this exploratory study justify larger-scale studies.

\section{Introduction}

Despite current well-developed standard of care regarding motor and sensorimotor rehabilitation in the United States, about $70 \%$ of adults with stroke (+/- 7 million Americans) still are unable to use their affected hand in daily life and live on with significant disability for many years [1-14], calling for more effective therapies to restore motor function, especially for those with severe motor impairments[13].

Cognitive multisensory rehabilitation $(\mathrm{CMR})[15-17]$ is a sensorimotor rehabilitation approach, in which the patient is guided to solve sensory discrimination exercises with eyes closed or to solve multisensory discrimination exercises (e.g., compare feeling shapes with seeing shapes) with eyes closed (Fig. 1). Other discrimination exercises encompass discriminating features of small objects such as distance, weight, resistance, texture discrimination etc. The key distinction between CMR and other sensorimotor rehabilitation approaches is that the CMR therapist asks the patient to reflect on how the limb was moved or was positioned, and how the movement was felt in the body, and to pay attention to how the limb movements are related to other parts of the body and spatial parameters in the environment. This distinct feature of reflective focus on the body might be the reason why adults with acute stroke and with severe motor impairments had better motor recovery after CMR than after standard motor rehabilitation, and why adults with chronic stroke showed significantly better motor outcomes after CMR than after taskoriented training as well[18,19], pointing to an effectiveness of CMR for motor recovery for adults with chronic stroke. Yet, the key question is: What is the underlying brain mechanism behind CMR? 
To answer this question, we previously identified brain areas that were activated during one of the CMR exercises, i.e. shape discrimination, in healthy adults and adolescents, adults with stroke and children with cerebral palsy using our custom-build MRI-compatible robot [20-23]. Based on our carefully controlled trials, we found that the cognitive processing of discriminating shapes -i.e. creating a mental picture of the first shape, keeping it in working memory to compare the distinct features of the first shape with the second shape- activated fronto-parietal brain areas, including the parietal operculum (parts OP1/OP4, also known as the secondary somatosensory cortex, SII), insula, and crus I/II of the cerebellum[20-23].

Interestingly, those crucial brain areas (i.e., OP1/OP4, insula and Crus I/II) were activated only when participants performed such cognitive processing or paid attention to the movement $[20,23]$. This discovery was significant because it revealed that OP1/OP4 might be preferentially activated when mindful attention is given to the movement.

As indicated by recent resting-state functional connectivity (FC) studies in healthy adults, OP1 and OP4 are an important part of the multimodal integration network in which visual, somatosensory, and auditory information are integrated to form visuospatial body maps in the posterior parietal cortex (PPC) [24-27]. The visuospatial maps are then used to guide and control motor actions [24,25]. Therefore, it is possible that CMR could influence the multimodal integration network via OP1/OP4.

Not surprisingly, deficits in any step of the sensorimotor integration pathway contribute to motor function deficits post-stroke [28]. Indeed, adults with stroke who have motor impairments can have alterations in brain connectivity at all stages of this multimodal integration process, from primary sensory areas to associative multisensory regions (e.g., PPC)[29].

All these observations led to the idea that CMR might elicit its effect on motor recovery post-stroke through improving connectivity within the multimodal integration network. More specifically, we hypothesized that CMR might restore resting-state FC between OP1/OP4 and other parts in the brain. To explore this idea, we conducted the pilot study to see (i) if and how OP1/OP4 connectivity is reduced post-stroke compared to healthy adults; and (ii) if CMR restores affected OP1/OP4 connectivity alongside recovery of sensorimotor function in adults with chronic stroke and upper limb motor impairments.

\section{Results}

\subsection{Demographics and behavioural findings}

Twenty-six healthy participants were contacted through convenience sampling: two had claustrophobia, two did not participate for medical reasons. Of the 14 screened participants with chronic stroke, 6 were excluded: 5 because of multiple brain lesions or location outside of the middle cerebral artery region; 1 because this participant received botox at the beginning of the study. Fig. 2 displays the flow chart. In sum, twenty-two healthy adults (average age $59 \pm 16$ years, range $30-84$ years, 14 women) and eight participants with chronic stroke (average age $53 \pm 14$ years, range $28-73$ years, 3 women, time since 
stroke $4 \pm 2.69$, range 1-7 years), all with infarct with a lesion in the right middle cerebral artery area and with resulting left hemiplegia/hemiparesis participated in the study. The demographic and clinical data of both the healthy participants and participants with stroke are presented in Table 1. Healthy adults were tested at baseline only.

Regarding general baseline data (Table 1), only education $(p=0.0006)$ and the Raven test $(p=0.01)$ were significantly lower in adults with stroke compared to healthy adults; other comparisons were not significantly different between both groups. As expected, stroke specific scales such as motor function (MESUPES arm function $p<0.0001$; hand function $p=0.0002$, total score $p=0.0002)$, apraxia $(p=0.02)$, exteroception $(p=0.0006)$, proprioception $(p=0.02)$, two-point discrimination $(p=0.02)$, and stereognosis $(p<0.0001)$ were significantly impaired in stroke at baseline compared to healthy adults.

The clinical data in patients with stroke at 3 time points (baseline, post-CMR, and at 1-year follow-up) with statistical results from the repeated measures ANOVA are presented in Supplementary Table S1, and we specified which tests were acquired on 8 or 4 patients.

The MESUPES arm motor function $(p=0.03)$, hand function $(p=0.03)$ and total score $(p=0.003)$ as well as Fugl-Meyer upper limb total score $(p=0.006)$ improved significantly between pre- and post-CMR and the improvement was maintained at 1-year follow-up. Our MESUPES assessments demonstrated that adults with stroke improved on average $22 \%$ in arm function and $39 \%$ in hand function, with retained benefit of $21 \%$ and $33 \%$, respectively, at 1-year follow-up compared to pre-CMR.

Stereognosis ( $p=0.03$ ) and Frenchay test $(p=0.03)$ improved significantly from baseline to 1-year followup. The Jebsen scores in four patients were not significantly different over time. We displayed in Fig. 3 the six individual Jebsen tasks with average speed for each task execution at the three time points. The four other patients could not perform the Jebsen tasks because their hand function was too severely impaired, rendering them unable to grasp the objects and perform the dexterity tasks. Of note is that the sensorimotor benefits were found after all patients had completed their standard rehabilitation care in the clinic and as outpatients.

GROC at 1-year follow-up are displayed per patient in Fig. 4. The patients reported GROC scores regarding their upper limb recovery after therapy between 2 (i.e., a little bit better) and 7 (i.e., a very great deal better).

\subsection{Resting-state connectivity in healthy adults and adults with stroke}

The resting-state functional analysis showed decreased FC between the right OP1/OP4 (lesioned hemisphere) and 34 areas distributed across all lobes in adults with stroke compared to healthy adults. After CMR, only four of those areas were significantly reduced in stroke compared to healthy adults: the right temporal pole, left cuneal cortex, left parietal operculum and left Heschl's gyrus (Supplementary

Table S2). Fig. 5 depicts a comparison between stroke and healthy adults in terms of connectivity before and after CMR. The regions from the Harvard-Oxford cortical atlas were used to demonstrate statistically 
significantly greater functional connectivity with the OP1/OP4 seed region (FDR-adjusted $p$-values $<0.05$ ) in healthy controls compared to the stroke group pre-CMR $(A)$ and in healthy controls versus the stroke group post-CMR (B).

Further, as an illustration, Fig. 6 depicts boxplots for the healthy controls, and for pre-CMR, post-CMR in stroke. The boxplots show significantly decreased FC between the right OP1/OP4 and two brain areas pre-CMR when adults with stroke were compared to healthy controls. However, after CMR, there was no statistically significant difference in strength in connectivity between adults with stroke and healthy controls. The test statistics and $p$-values are: $(\mathrm{A})$ the right OP1/OP4 and the right postcentral gyrus (i.e., stroke pre-CMR $t$-statistic $=3.08$, stroke $<$ healthy: adjusted $p=0.013$; stroke post-CMR $t$-statistic $=2.72$, stroke < healthy: adjusted $p=0.076$ ), and (B) the right OP1/OP4 and the right superior parietal lobe (i.e., stroke pre-CMR $t$-statistic $=2.56$, stroke $<$ healthy: adjusted $p=0.021$; stroke post-CMR $t$-statistic $=0.08$, stroke $<$ healthy: adjusted $p=0.938$ ).

\section{Discussion}

Our behavioural results regarding motor function and participation in daily activities are in alignment with the previously reported CMR studies in patients with acute and chronic stroke, $[18,19]$ pointing to validity of our pilot clinical study regarding the effect of CMR on behavioural outcomes.

To the best of our knowledge, this is the first brain imaging study, which explored brain connectivity changes related to CMR. These exploratory results support the concept that, after a stroke, neurorehabilitation can target neuroplasticity by increasing connectivity in sensory and motor networks, $[30,31]$ and expands further knowledge on recovery of sensory networks following rehabilitation.

In this study, we observed that, after CMR, many of the 30 restored connections were connections between OP1/OP4 and other areas within the multisensory integration network ( Fig. 7 ). This network provides essential information in order to generate and perform accurate motor actions. [26,27,32-35] Therefore, it is likely that restoration of connections within this network that includes OP1/OP4 led to recovery of motor function. Furthermore, the importance of OP1/OP4 for their potential role in motor recovery is strengthened by the finding that OP1/OP4 activity measured during acute stroke appears to be a good indicator of how well sensorimotor could be recovered 6 months later in adults with large hemispheric strokes and moderate to severe motor impairments [36]. How exactly activation of OP1/OP4 leads to restoration of connectivity in the brain relevant for motor function remains unknown and will be subject of our future investigations.

The underlying mechanism of how the cognitive processing, reflection, and attention to sensorimotor tasks during CMR influences motor recovery also remains largely unknown and is the main subject of our ongoing investigation. Nonetheless, the current understanding of how the integration of multisensory information influences motor function at the brain level, as illustrated in Fig. 7 , may provide a clue as to how CMR may function to restore motor function. 
In CMR exercises, the therapist will probe the patient through questions to consciously reflect on the position of their arm and hand in space and to have a focused awareness to the multisensory processing and their movements during sensory discrimination exercises. In the end, the CMR exercises performed by patients probably help them recalibrate what is called their "mental body representations" (MBR). MBR refers to accurate updated information about body positions and movements in space at any moment, and thus MBR is critical to performing motor actions correctly $[37,38]$. Additionally, recent literature has shown that $81 \%$ of adults with vascular brain injuries exhibit deficits in at least one type of MBR [39] .

How MBR may fit into the current understanding of the multi-sensorimotor cycle in the brain is illustrated in Fig. 7 . Our proposed model in Fig. 7 is constructed based on the multimodal integration network model of Sepulcre et al. $(2014 a, 2014 b)$ in healthy adults [26,27] ; on our previous work [20-23] ; and on the work of others $[24,33,40-45]$. In this model, information from the primary sensory and motor cortex reaches the multimodal integration network via OP4, where sensory, motor and visual information are all converged $[26,27]$ to form body awareness, also called body schema $[24,33,40-45]$. Body awareness in this context, refers to a perceptual understanding and awareness of (i) proprioception: body position and movement sense and how those body parts are situated in peripersonal space, (ii) exteroception: visual, tactile, auditory signals locations within the peripersonal space; (iii) interoception: internal body states $[24,33,40-45]$. Then, the information formed in the multimodal integration network is sent to the posterior PPC in which the body image is formed [38,46] . Body image refers to dynamic visuospatial body maps that house a cognitive understanding and awareness of how the whole body is situated in space at each timepoint $[38,39,46,47]$. Body awareness and body image are two different types of MBR. According to Caggiano and Cocchini (2020) and Pitron et al . (2018), body awareness and body image interact with each other $[48,49]$. Finally, the body image is necessary to guide execution of precise motor actions, such as hand movements ( Fig. 7 ) [37] .

Considering this model, CMR exercises are targeting the restoration of body awareness directly, which in turn, directly and indirectly improves body image and thus MBR as a whole, which aids restoration of motor function. At the brain function level, we speculate that (i) OP1/OP4 in the multimodal integration network plays a crucial role in the formation of an accurate body awareness, and that (ii) improvement of body awareness by CMR may activate OP1/OP4, leading to restoration of the brain connectivity that we have observed.

The presented data justify future large scale studies, which investigate changes in brain activation and connections related to CMR exercise-specific activation in patients with stroke compared to healthy volunteers, as well as investigating more broadly brain changes before and after CMR treatment in relation to sensorimotor recovery in a larger sample of adults with stroke.

\section{Methods}

\subsection{Participants}


All participants were recruited between November 2015 and August 2017. The participants signed an informed consent and the study was in accordance with the Declaration of Helsinki. The Institutional Review Board (IRB) of the University of Minnesota approved the study.

Adults with stroke were included if they (i) were between 18-85 years old, (ii) were at least 6 months after a stroke that occurred in adulthood, (ii) had a stable ischemic infarct with a lesion in the right middle cerebral artery area, with resulting left hemiplegia/paresis, (iv) did not receive ongoing rehabilitation at the time of testing, (v) were able to read, hear and comprehend instructions in English. Exclusion criteria were: (i) contra-indications for Magnetic Resonance Imaging (MRI) scanning; (ii) other medical conditions precluding full participation, including (other) brain injuries/illnesses, cognitive impairment, severe unilateral spatial neglect or severe proprioceptive loss, hindering them to feel finger movements on the affected side, severe apraxia, severe aphasia, or contractures that restricts them from keeping the outstretched arm in a relaxed position.

Healthy adults of similar age than adults with stroke were recruited through fliers and through the University website. Healthy participants had to be medically stable; able to read, hear and comprehend instructions in English. We excluded healthy adults who had brain injuries, who had complete proprioceptive loss, contractures in the arm, who were medically unstable, illiterate in English, having cognitive impairments, or for whom MRI scanning was contra-indicated.

\subsection{Research design}

This is an exploratory pilot study with single arm pre-post experimental design in which participants with stroke were tested with behavioural tests, structural and resting-state functional MRI at baseline; behavioural test, structural and resting-state functional MRI after 6 weeks of therapy (CMR, 3 times a week for $45 \mathrm{~min} /$ session), and behavioural tests at follow-up, 1 year after the therapy has ended. Healthy volunteers were recruited as a control group and were tested once on behavioural tests, structural and resting-state functional MRI.

\subsection{Clinical assessments}

All participants (i.e. healthy adults as well as adults with stroke) underwent screening tests, i.e., Bell's test for neglect (cut-off <29/35)[50], Mini-Mental State Examination-short version MMSE $®$-2-BV ${ }^{\text {TM }}$ (cut-off $<13 / 16$ )[51], Apraxia Screen test (cut-off $<5 / 12$ for severe apraxia),[52] Aphasia Rapid Test [cut-off $>21 / 26$ (lower score is better outcome)],[53] Edinburgh Handedness Inventory[54], the National Institutes of Health Stroke Scale (NIHSS)[55], Raven's Progressive Matrices to measure abstract reasoning,[56] and the Corsi Block Tapping Task[57] to test visuo-spatial short-term memory, exteroceptive and proprioceptive sensibility (tested clinically for position and motion sense), two-point discrimination, in stereognosis, in which participants need to correctly identify objects by feeling the object in the hand with eyes closed,[58] and Numeric Rating scale[59] for pain in upper limb. Participants also completed medical and general health questionnaires. 
Motor function of the affected arm was evaluated with the "Motor Evaluation for Upper Extremity in Stroke Patients" (MESUPES) in which scores are given for the perceived muscle tone during passive arm movements; for assisted and active arm movements, and for active hand and finger movements, including dexterity tasks (e.g., rotating a dice with thumb and index finger)[14,60]. Secondary clinical measures were the Jebsen-Taylor Hand Function Test[61], which tests hand dexterity (such as putting beans in a contained with a spoon); Fugl-Meyer upper extremity test[62]; ABILHAND in which participants reflect on how easily daily bimanual tasks can performed[63]; Stroke Impact Scale[64]; EuroQol-5D-5L[65]; Global rating scale of change[66]; Frenchay test[67], which enquires how often participants are engaged in daily activities; and the Warwick-Edinburgh Mental Well-Being Scale.[68] The global rating of change scale (GROC) evaluates the patient's grading of stroke recovery in terms of upper limb motor function after therapy, ranging from -7 (i.e., a very great deal worse) to 7 (i.e., a very great deal better)[66].

\subsection{Cognitive multisensory rehabilitation (CMR)}

CMR, whose original Italian name is "riabilitazione neurocognitiva", is also translated in other publications as cognitive therapeutic exercises[69], Cognitive Sensory Motor Training[19], neurocognitive therapeutic exercise[70], cognitive exercise therapy[18], or (neuro)cognitive approach[71,72].

CMR incorporates conscious perception of body positions and movements during (multi)sensory discrimination exercises[15-17]. The treating therapists, with years of clinical practice and specialized in this therapy, gave 35 minutes of discrimination exercises embedded in functional movements followed by 10 minutes of applying the learned strategies during activities of daily living. The difficulty of the exercises was set at $+/-75 \%$ success, monitored by the number of correct responses given to an exercise, to promote learning, engage motivation and avoid frustration. CMR uses several types of discrimination exercises: Participants discriminated shapes, length, weight, distance, resistance, textures or compare kinaesthetic information with visual information for integration of multisensory information $[9,16]$. Solving the discrimination task is combined with reflection and a learning process, prompted by the therapist on how the limb (was) moved or was positioned. Focusing attention on movements helped participants control muscle tone and relax their hand,[73,74] thereby creating the potential for movements to re-emerge. Exercises focused on activating only those muscles relevant for the movement, adapting a correct relaxed sensation of the movement in proper form; relate and compare movement with the affected and unaffected arm; understanding the different movement components and how they relate to each other; and recognizing spatial and temporal cues.

Furthermore, kinaesthetic motor imagery was included to increase correct sense perception and, if present, to decrease pain[17,70]. Depending on the severity of the upper limb motor impairment, exercises transitioned from passive, assisted, active, and to functional movements progressively throughout the therapy. All activities were directly related to functional tasks. Several body joints were included in the exercise to integrate speed and dexterity in functional movements.

\subsection{Structural and resting-state functional MRI (fMRI) acquisition and pre-processing}


Structural MRI acquisition was acquired using a T1 weighted MPRAGE image [TR=2.5s, TE=3.65ms, $1 \mathrm{~mm}^{3}$ voxels], as well as $\mathrm{T} 2$ weighted SPACE image [TR=3s, TE $=565 \mathrm{~ms}, 1 \mathrm{~mm}^{3}$ voxels], and SPACE based FLAIR [TR=5.0s, TE=394ms, $1 \mathrm{~mm}^{3}$ voxels] images to quantify lesion extent. The 3T Siemens auto-align longitudinal repositioning system was used to ensure comparable head positioning across the scan sessions. Structural Preprocessing was performed using the containerized FMRIPREP[75] version 1.2.2 with standardized BIDS formatted NIFTI data.[76] Each T1-weighted (T1w) volume was corrected for intensity non-uniformity (INU) using N4BiasFieldCorrection[77] and skull-stripped using antsBrainExtraction.sh using the OASIS template. Brain surfaces were reconstructed using recon-all from FreeSurfer,[78] and the brain mask estimates were refined with a custom variation of the method to reconcile ANTs-derived and FreeSurfer-derived segmentations of the cortical gray-matter of Mindboggle. [79] Spatial normalization to the ICBM 152 Nonlinear Asymmetrical template[80] was performed through nonlinear registration with the antsRegistration tool of ANTs,[81] using brain-extracted versions of both T1w volume and template. Additionally, for participants with focal brain lesions, a lesion mask was added during spatial normalization to standard space.[82] Brain tissue segmentation of cerebrospinal fluid (CSF), white-matter (WM) and gray-matter (GM) was performed on the brain-extracted T1w using FAST.[83]

Resting-state functional connectivity (FC) is a measure of the temporal correlation of activation between spatially separate brain regions.[84] fMRI acquisition for resting-state was obtained with a T2*-weighted multiband echo planar acquisition tipped 30 degrees relative to the AC-PC plane. This sequence was designed to measure whole-head BOLD-contrast with optimal temporal and spatial resolution, and to reduce signal dropout [TR=0.871s; TE=34.20ms; Flip Angle=65deg; 72 slices; multiband factor $6 ; 2.20 \mathrm{~mm}$ isotropic resolution]. fMRI Preprocessing was also performed with the containerized FMRIPREP[85] 1.2.2 version with standardized BIDS formatted NIFTI data.[76] First, a reference volume and its skull-stripped version were generated using a custom methodology of FMRIPREP. Functional data were slice time corrected using 3dTshift from AFNI[86] and motion corrected using mcflirt.[87] Distortion correction was performed using an implementation of the TOPUP technique[88] using 3dQwarp.[86] This was followed by co-registration to the corresponding T1w using boundary-based registration[89] with 9 degrees of freedom, using bbregister (FreeSurfer). Motion correcting transformations, field distortion correcting warp, BOLD-to-T1w transformation and T1w-to-template ( $\mathrm{MNI}$ ) warp were concatenated and applied in a single step using antsApplyTransforms (ANTs v2.1.0) using Lanczos interpolation. Physiological noise regressors were extracted applying CompCor.[90] Principal components were estimated for the two CompCor variants: temporal (tCompCor) and anatomical (aCompCor). A mask to exclude signal with cortical origin was obtained by eroding the brain mask, ensuring it only contained subcortical structures. Six tCompCor components were then calculated, including only the top $5 \%$ variable voxels within that subcortical mask. For aCompCor, six components were calculated within the intersection of the subcortical mask and the union of CSF and WM masks calculated in T1w space, after their projection to the native space of each functional run. Framewise displacement and DVARS[91] were calculated for each functional run using the implementation of Nipype. ICA-based Automatic Removal Of Motion 
Artifacts (AROMA) were used to generate aggressive noise regressors and to create a variant of data that was non-aggressively denoised.[92]Resting-state fMRI: set-up and statistical analysis

Participants underwent a resting-state fMRI scan of 14.50 minutes with eyes open, maintaining fixation with a restful mind and they were asked to not fall asleep. The Harvard-Oxford cortical atlas was split into regions corresponding to each of the left and right hemisphere, resulting in a total of 96 regions of interest (ROI). The resulting resting-state BOLD-contrast time series were extracted from each $\mathrm{ROI}$ from each participant by averaging the voxel-level time courses within each ROI. For each participant, Pearson correlation coefficients were computed to construct functional connectivity metrics between the OP1/OP4 in the affected (i.e., right) hemisphere, thereby serving as the seed region in the analysis and the other ROls. Two-sample $t$-tests were used to determine differences between the participants with stroke and healthy controls in the FC between the seed region and each ROI. The Benjamini-Hochberg procedure for controlling the false discovery rate (FDR) was used to adjust the $p$-values to account for multiple comparisons.

\subsection{Behavioural data: Statistical analysis}

Behavioural data were calculated with JMP®, Version 13, SAS Institute Inc., Cary, NC, 1989-2007. The results of the Shapiro-Wilk test to verify normal distribution of ratio data informed the decision to conduct two-sample $t$-tests or Mann-Whitney $U$ tests to compare both groups at baseline. Nominal data were calculated with Chi square (test of independence) between groups (https://www.socscistatistics.com/tests/chisquare/Default2.aspx). Repeated measures ANOVA was used to compare the clinical data in adults with stroke at baseline, after CMR, and at 1-year follow-up. Subgroup analyses for sex, race or ethnicity were not feasible due to the small sample size.

\section{Declarations}

\section{Acknowledgements}

We would like to thank Dr. Yuichiro Takagi for the critical reading of the manuscript. This work was supported by the OVPR Grant-in-Aid of Research, Artistry, and Scholarship program University of Minnesota (\#110760), The National Institutes of Health (NIH) P41 EB015894, P30 NS076408 “Institutional Centre Cores for Advanced Neuroimaging", 1S100D017974-01 "High Performance Connectome Upgrade for Human 3T MR Scanner", and the National Centre for Advancing Translational Sciences of the National Institutes of Health Award Number UL1-TR002494. The content is solely the responsibility of the authors and does not necessarily represent the official views of the National Institutes of Health. None of the funding agencies played a role in the design, execution, analysis, data interpretation or writing of the manuscript.

\section{Author Contributions}


AVDW, DDP, MR, BAM, CS, FI, and KOL contributed conception and design of the study; AVDW, ML, BDJ, $\mathrm{WE}, \mathrm{TH}$, and MF organized the database; TH, MF, and KOL performed the statistical analysis; AVDW wrote the first draft of the manuscript; TH, MF, DDP, and MR wrote sections of the manuscript. All authors contributed to manuscript revision and have read and approved the submitted version.

\section{Competing Interest Statement}

The authors declare no competing interests.

\section{Data Availability Statement}

The datasets generated during and analysed during the current study are available from the corresponding author on reasonable request.

\section{References}

1. Aman, J. E., Elangovan, N., Yeh, I. L. \& Konczak, J. The effectiveness of proprioceptive training for improving motor function: a systematic review. Front. Hum. Neurosci. 8, 1075 (2014).

2. Byl, N. N., Pitsch, E. A. \& Abrams, G. M. Functional outcomes can vary by dose: learning-based sensorimotor training for patients stable poststroke. Neurorehabil. Neural Repair 22, 494-504 (2008).

3. Edwards, L. L., King, E. M., Buetefisch, C. M. \& Borich, M. R. Putting the 'Sensory' Into Sensorimotor Control: The Role of Sensorimotor Integration in Goal-Directed Hand Movements After Stroke. Frontiers in Integrative Neuroscience vol. 13 (2019).

4. Wolf, S. L. et al. Effect of constraint-induced movement therapy on upper extremity function 3 to 9 months after stroke: the EXCITE randomized clinical trial. JAMA 296, 2095-2104 (2006).

5. Kwakkel, G., Veerbeek, J. M., van Wegen, E. E. H. \& Wolf, S. L. Constraint-induced movement therapy after stroke. Lancet Neurol. 14, 224-234 (2015).

6. Lang, C. E. et al. Dose response of task-specific upper limb training in people at least 6 months poststroke: A phase II, single-blind, randomized, controlled trial. Ann. Neurol. 80, 342-354 (2016).

7. Schuster-Amft, C. et al. Effect of a four-week virtual reality-based training versus conventional therapy on upper limb motor function after stroke: A multicenter parallel group randomized trial. PLOS ONE vol. 13 e0204455 (2018).

8. Blennerhassett, J. M., Matyas, T. A. \& Carey, L. M. Impaired discrimination of surface friction contributes to pinch grip deficit after stroke. Neurorehabil. Neural Repair 21, 263-272 (2007).

9. Carey, L., Macdonell, R. \& Matyas, T. A. SENSe: Study of the Effectiveness of Neurorehabilitation on Sensation: a randomized controlled trial. Neurorehabil. Neural Repair 25, 304-313 (2011).

10. Chen, X. et al. Therapeutic effects of sensory input training on motor function rehabilitation after stroke. Medicine 97, e13387 (2018). 
11. Turville, M. L., Cahill, L. S., Matyas, T. A., Blennerhassett, J. M. \& Carey, L. M. The effectiveness of somatosensory retraining for improving sensory function in the arm following stroke: a systematic review. Clin. Rehabil. 33, 834-846 (2019).

12. Benjamin, E. J. et al. Heart Disease and Stroke Statistics-2019 Update: A Report From the American Heart Association. Circulation 139, e56-e528 (2019).

13. Cramer, S. C. et al. Stroke Recovery and Rehabilitation Research: Issues, Opportunities, and the National Institutes of Health StrokeNet. Stroke 48, 813-819 (2017).

14. Van de Winckel, A. et al. Can quality of movement be measured? Rasch analysis and inter-rater reliability of the Motor Evaluation Scale for Upper Extremity in Stroke Patients (MESUPES). Clin. Rehabil. 20, 871-884 (2006).

15. Perfetti, C. Der hemiplegische patient. Kognitiv-therapeutische übungen. (1997).

16. Perfetti, C. L'exercice thérapeutique cognitif pour la rééducation du patient hémiplégique. vol. 12 (2001).

17. Perfetti, C. et al. Il dolore come problema riabilitativo. (Piccin, 2015).

18. Lee, S., Bae, S., Jeon, D. \& Kim, K. Y. The effects of cognitive exercise therapy on chronic stroke patients' upper limb functions, activities of daily living and quality of life. J. Phys. Therapy Sci. 27, 2787-2791 (2015).

19. Chanubol, R. et al. A randomized controlled trial of Cognitive Sensory Motor Training Therapy on the recovery of arm function in acute stroke patients. Clin. Rehabil. 26, 1096-1104 (2012).

20. Van de Winckel, A. et al. How does brain activation differ in children with unilateral cerebral palsy compared to typically developing children, during active and passive movements, and tactile stimulation? An fMRI study. Research in Developmental Disabilities vol. 34 183-197 (2013).

21. Van de Winckel, A. et al. Passive somatosensory discrimination tasks in healthy volunteers: differential networks involved in familiar versus unfamiliar shape and length discrimination. Neuroimage 26, 441-453 (2005).

22. Van de Winckel, A. et al. Frontoparietal involvement in passively guided shape and length discrimination: a comparison between subcortical stroke patients and healthy controls. Exp. Brain Res. 220, 179-189 (2012).

23. Van de Winckel, A. et al. Does somatosensory discrimination activate different brain areas in children with unilateral cerebral palsy compared to typically developing children? An fMRI study. Res. Dev. Disabil. 34, 1710-1720 (2013).

24. Park, H.-D. \& Blanke, O. Coupling Inner and Outer Body for Self-Consciousness. Trends Cogn. Sci. 23, 377-388 (2019).

25. Bretas, R. V., Taoka, M., Suzuki, H. \& Iriki, A. Secondary somatosensory cortex of primates: beyond body maps, toward conscious self-in-the-world maps. Exp. Brain Res. 238, 259-272 (2020).

26. Sepulcre, J. Functional streams and cortical integration in the human brain. Neuroscientist 20, 499508 (2014). 
27. Sepulcre, J. Integration of visual and motor functional streams in the human brain. Neurosci. Lett. 567, 68-73 (2014).

28. Bolognini, N., Russo, C. \& Edwards, D. J. The sensory side of post-stroke motor rehabilitation. Restor. Neurol. Neurosci. 34, 571-586 (2016).

29. Grefkes, C. \& Fink, G. R. Reorganization of cerebral networks after stroke: new insights from neuroimaging with connectivity approaches. Brain 134, 1264-1276 (2011).

30. Fan, Y. T. et al. Neuroplastic changes in resting-state functional connectivity after stroke rehabilitation. Front. Hum. Neurosci. 9, 546 (2015).

31. Sharma, N., Baron, J. C. \& Rowe, J. B. Motor imagery after stroke: relating outcome to motor network connectivity. Ann. Neurol. 66, 604-616 (2009).

32. Choi, W., Li, L., Satoh, S. \& Hachimura, K. Multisensory Integration in the Virtual Hand Illusion with Active Movement. Biomed Res. Int. 2016, 8163098 (2016).

33. Daprati, E., Sirigu, A. \& Nico, D. Body and movement: consciousness in the parietal lobes. Neuropsychologia 48, 756-762 (2010).

34. Palermo, L. et al. Action and Non-Action Oriented Body Representations: Insight from Behavioural and Grey Matter Modifications in Individuals with Lower Limb Amputation. Biomed Res. Int. 2018, 1529730 (2018).

35. Sepulcre, J., Sabuncu, M. R., Yeo, T. B., Liu, H. \& Johnson, K. A. Stepwise connectivity of the modal cortex reveals the multimodal organization of the human brain. J. Neurosci. 32, 10649-10661 (2012).

36. Hannanu, F. F. et al. Parietal operculum and motor cortex activities predict motor recovery in moderate to severe stroke. Neuroimage Clin 14, 518-529 (2017).

37. Oouchida, Y. et al. Maladaptive change of body representation in the brain after damage to central or peripheral nervous system. Neurosci. Res. 104, 38-43 (2016).

38. Whitlock, J. R. Posterior parietal cortex. Curr. Biol. 27, R691-R695 (2017).

39. Razmus, M. Body representation in patients after vascular brain injuries. Cogn. Process. 18, 359-373 (2017).

40. Garfinkel, S. N. et al. Interoceptive dimensions across cardiac and respiratory axes. Philos. Trans. R. Soc. Lond. B Biol. Sci. 371, (2016).

41. Dijkerman, H. C. \& de Haan, E. H. Somatosensory processes subserving perception and action. Behav. Brain Sci. 30, 189-201; discussion 201-39 (2007).

42. Khalsa, S. S. et al. Interoception and Mental Health: A Roadmap. Biol Psychiatry Cogn Neurosci Neuroimaging 3, 501-513 (2018).

43. Mehling, W. E. et al. Body Awareness: a phenomenological inquiry into the common ground of mindbody therapies. Philos. Ethics Humanit. Med. 6, 6 (2011).

44. Young, K. S. et al. The impact of mindfulness-based interventions on brain activity: A systematic review of functional magnetic resonance imaging studies. Neurosci. Biobehav. Rev. 84, 424-433 
(2018).

45. Gibson, J. Mindfulness, Interoception, and the Body: A Contemporary Perspective. Front. Psychol. 10, 2012 (2019).

46. Berlucchi, G. \& Vallar, G. The history of the neurophysiology and neurology of the parietal lobe. in Handbook of clinical neurology vol. 151 3-30 (Elsevier, 2018).

47. de Vignemont, F. Body schema and body image-Pros and cons. Neuropsychologia 48, 669-680 (2010).

48. Caggiano, P. \& Cocchini, G. The functional body: does body representation reflect functional properties? Experimental Brain Research vol. 238 153-169 (2020).

49. Pitron, V., Alsmith, A. \& de Vignemont, F. How do the body schema and the body image interact? Conscious. Cogn. 65, 352-358 (2018).

50. Gauthier, L., Dehaut, F. \& Joanette, Y. The bells test: a quantitative and qualitative test for visual neglect. International Journal of Clinical Neuropsychology (1989).

51. Folstein, M. F., Folstein, S. E. \& McHugh, P. R. 'Mini-mental state': a practical method for grading the cognitive state of patients for the clinician. J. Psychiatr. Res. 12, 189-198 (1975).

52. Vanbellingen, T. et al. Comprehensive assessment of gesture production: a new test of upper limb apraxia (TULIA). Eur. J. Neurol. 17, 59-66 (2010).

53. Azuar, C. et al. The Aphasia Rapid Test: an NIHSS-like aphasia test. J. Neurol. 260, 2110-2117 (2013).

54. Oldfield, R. C. The assessment and analysis of handedness: the Edinburgh inventory. Neuropsychologia 9, 97-113 (1971).

55. Kwah, L. K. \& Diong, J. National Institutes of Health Stroke Scale (NIHSS). J. Physiother. 60, 61 (2014).

56. van den Berg, E. et al. Exploration of the Raven APM-National Adult Reading Test discrepancy as a measure of intellectual decline in older persons. Appl. Neuropsychol. Adult 20, 7-14 (2013).

57. Kessels, R. P., van Zandvoort, M. J., Postma, A., Kappelle, L. J. \& de Haan, E. H. The Corsi BlockTapping Task: standardization and normative data. Appl. Neuropsychol. 7, 252-258 (2000).

58. Klingels, K. et al. A systematic review of arm activity measures for children with hemiplegic cerebral palsy. Clin. Rehabil. 24, 887-900 (2010).

59. Safikhani, S. et al. Response scale selection in adult pain measures: results from a literature review. $J$ Patient Rep Outcomes 2, 40 (2017).

60. Johansson, G. M. \& Häger, C. K. Measurement properties of the Motor Evaluation Scale for Upper Extremity in Stroke patients (MESUPES). Disabil. Rehabil. 34, 288-294 (2012).

61. Jebsen, R. H., Taylor, N., Trieschmann, R. B., Trotter, M. J. \& Howard, L. A. An objective and standardized test of hand function. Arch. Phys. Med. Rehabil. 50, 311-319 (1969).

62. Fugl-Meyer, A. R., Jääskö, L., Leyman, I., Olsson, S. \& Steglind, S. The post-stroke hemiplegic patient. 1. a method for evaluation of physical performance. Scand. J. Rehabil. Med. 7, 13-31 (1975). 
63. Penta, M., Tesio, L., Arnould, C., Zancan, A. \& Thonnard, J. L. The ABILHAND questionnaire as a measure of manual ability in chronic stroke patients: Rasch-based validation and relationship to upper limb impairment. Stroke 32, 1627-1634 (2001).

64. Lin, K. C. et al. Psychometric comparisons of the Stroke Impact Scale 3.0 and Stroke-Specific Quality of Life Scale. Qual. Life Res. 19, 435-443 (2010).

65. Dorman, P. J., Waddell, F., Slattery, J., Dennis, M. \& Sandercock, P. Is the EuroQol a valid measure of health-related quality of life after stroke? Stroke 28, 1876-1882 (1997).

66. Kamper, S. J., Maher, C. G. \& Mackay, G. Global rating of change scales: a review of strengths and weaknesses and considerations for design. J. Man. Manip. Ther. 17, 163-170 (2009).

67. Wade, D. T., Langton-Hewer, R., Wood, V. A., Skilbeck, C. E. \& Ismail, H. M. The hemiplegic arm after stroke: measurement and recovery. J. Neurol. Neurosurg. Psychiatry 46, 521-524 (1983).

68. Stewart-Brown, S. et al. Internal construct validity of the Warwick-Edinburgh Mental Well-being Scale (WEMWBS): a Rasch analysis using data from the Scottish Health Education Population Survey. Health Qual. Life Outcomes 7, 15 (2009).

69. De Patre, D. et al. Visual and Motor Recovery After 'Cognitive Therapeutic Exercises' in Cortical Blindness: A Case Study. J. Neurol. Phys. Ther. 41, 164-172 (2017).

70. Marzetti, E. et al. Neurocognitive therapeutic exercise improves pain and function in patients with shoulder impingement syndrome: a single-blind randomized controlled clinical trial. Eur. J. Phys. Rehabil. Med. 50, 255-264 (2014).

71. Morreale, M. et al. Early versus delayed rehabilitation treatment in hemiplegic patients with ischemic stroke: proprioceptive or cognitive approach? Eur. J. Phys. Rehabil. Med. 52, 81-89 (2016).

72. Sallés, L. et al. A neurocognitive approach for recovering upper extremity movement following subacute stroke: a randomized controlled pilot study. J. Phys. Therapy Sci. 29, 665-672 (2017).

73. Carey, L. M. Stroke Rehabilitation: Insights from Neuroscience and Imaging. Chapter 12:157-172. (Oxford University Press, 2012).

74. Balakrishnan, S. \& Ward, A. B. The diagnosis and management of adults with spasticity. Handb. Clin. Neurol. 110, 145-160 (2013).

75. Esteban, O. et al. fMRIPrep: a robust preprocessing pipeline for functional MRI. (2019). doi:10.5281/zenodo.2859286.

76. Gorgolewski, K. J. et al. The brain imaging data structure, a format for organizing and describing outputs of neuroimaging experiments. Scientific Data vol. 3160044 (2016).

77. Tustison, N. J. et al. N4ITK: improved N3 bias correction. IEEE Trans. Med. Imaging 29, 1310-1320 (2010).

78. Dale, A. M., Fischl, B. \& Sereno, M. I. Cortical surface-based analysis. I. Segmentation and surface reconstruction. Neuroimage 9, 179-194 (1999).

79. Klein, A. et al. Mindboggling morphometry of human brains. PLoS Comput. Biol. 13, e1005350 (2017). 
80. Fonov, V. S., Evans, A. C., McKinstry, R. C., Almli, C. R. \& Collins, D. L. Unbiased nonlinear average ageappropriate brain templates from birth to adulthood. Neuroimage 47, S102 (2009).

81. Avants, B. B., Epstein, C. L., Grossman, M. \& Gee, J. C. Symmetric diffeomorphic image registration with cross-correlation: evaluating automated labeling of elderly and neurodegenerative brain. Med. Image Anal. 12, 26-41 (2008).

82. Brett, M., Leff, A. P., Rorden, C. \& Ashburner, J. Spatial normalization of brain images with focal lesions using cost function masking. Neuroimage 14, 486-500 (2001).

83. Zhang, Y., Brady, M. \& Smith, S. Segmentation of brain MR images through a hidden Markov random field model and the expectation-maximization algorithm. IEEE Trans. Med. Imaging 20, 45-57 (2001).

84. Young, B. M. et al. Changes in functional connectivity correlate with behavioral gains in stroke patients after therapy using a brain-computer interface device. Front. Neuroeng. 7, 25 (2014).

85. Esteban, O. et al. fMRIPrep: a robust preprocessing pipeline for functional MRI. Nature Methods vol. $16111-116$ (2019).

86. Cox, R. W. AFNI: software for analysis and visualization of functional magnetic resonance neuroimages. Comput. Biomed. Res. 29, 162-173 (1996).

87. Jenkinson, M., Bannister, P., Brady, M. \& Smith, S. Improved optimization for the robust and accurate linear registration and motion correction of brain images. Neuroimage 17, 825-841 (2002).

88. Andersson, J. L. R., Skare, S. \& Ashburner, J. How to correct susceptibility distortions in spin-echo echo-planar images: application to diffusion tensor imaging. Neuroimage 20, 870-888 (2003).

89. Greve, D. N. \& Fischl, B. Accurate and robust brain image alignment using boundary-based registration. Neuroimage 48, 63-72 (2009).

90. Behzadi, Y., Restom, K., Liau, J. \& Liu, T. T. A component based noise correction method (CompCor) for BOLD and perfusion based fMRI. Neuroimage 37, 90-101 (2007).

91. Power, J. D. et al. Methods to detect, characterize, and remove motion artifact in resting state fMRI. Neuroimage 84, 320-341 (2014).

92. Pruim, R. H. R. et al. ICA-AROMA: A robust ICA-based strategy for removing motion artifacts from fMRI data. Neuroimage 112, 267-277 (2015).

\section{Tables}

Table 1. Demographic and screening data of healthy participants and adults with stroke 


\begin{tabular}{|c|c|c|c|}
\hline & $\begin{array}{l}\text { Healthy adults } \\
(n=22)\end{array}$ & $\begin{array}{l}\text { Adults with stroke } \\
(\mathrm{n}=8)\end{array}$ & Statistics \\
\hline Age (years, $X \pm S D$, range) & $59 \pm 16(30-84)$ & $53 \pm 14(28-73)$ & $t=-0.97, p=0.34$ \\
\hline Sex (females/males) & $14 / 8$ & $3 / 5$ & $\begin{array}{l}X^{2}=0.242 \\
p>0.05\end{array}$ \\
\hline Race (white/other) & $21 / 1$ & $7 / 1$ & $\begin{array}{l}X^{2}=0.469 \\
p>0.05\end{array}$ \\
\hline Ethnicity (not Hispanic/Hispanic) & $22 / 0$ & $8 / 0$ & $X^{2}=1, p>0.05$ \\
\hline $\begin{array}{l}\text { Edinburgh Handedness } \\
\text { (left/mixed/right) }\end{array}$ & $2 / 3 / 17$ & $0 / 0 / 8$ & \\
\hline Education (years, average $+/$-SD, range) & $18 \pm 2(14-21.5)$ & $15 \pm 2(12-17)$ & $\begin{array}{l}t=-3.86 \\
p=0.0006 *\end{array}$ \\
\hline $\begin{array}{l}\text { Corsi block span forwards } \\
\text { ( } 5 \text { or } 6 \text { is a normal score) }\end{array}$ & $6 \pm 1(5-9)$ & $6 \pm 2(5-9)$ & $\begin{array}{l}U=-0.08 \\
p=0.94\end{array}$ \\
\hline Corsi block span backwards & $6 \pm 1(4-9)$ & $6 \pm 2(4-8)$ & $\begin{array}{l}U=-1.25 \\
p=0.21\end{array}$ \\
\hline Raven (percentage, range) & $\begin{array}{l}69.38 \pm 27.16 \\
(0-99)\end{array}$ & $\begin{array}{l}40.25 \pm 24.08(14- \\
75)\end{array}$ & $\begin{array}{l}t=-2.66 \\
p=0.01 *\end{array}$ \\
\hline Bell's test (total scores 35) & $33.55 \pm 1.57$ & $34.25 \pm 1.39$ & $\begin{array}{l}U=1.26 \\
p=0.21\end{array}$ \\
\hline $\begin{array}{l}\text { MMSE-short version (total score } 16 \text {, } \\
\text { range) }\end{array}$ & $15 \pm 1(14-16)$ & $15 \pm 1(14-16)$ & $\begin{array}{l}U=-0.94 \\
p=0.34\end{array}$ \\
\hline Apraxia test (total score 12) & $12 \pm 0$ & $11 \pm 1$ & $\begin{array}{l}U=-2.38 \\
p=0.02 *\end{array}$ \\
\hline Aphasia rapid test ( 0 is a normal score) & $0 \pm 0$ & $0 \pm 1$ & $\begin{array}{l}U=1.15 \\
p=0.25\end{array}$ \\
\hline NIHSS & N/A & $3 \pm 1(n=4)$ & \\
\hline Numeric pain rating scale $(n=8)$ & $0.90 \pm 1.37$ & $1.88 \pm 3.40$ & $\begin{array}{l}U=0.48 \\
p=0.63\end{array}$ \\
\hline MESUPES arm L (total 40) $(n=8)$ & $40.00 \pm 0.00$ & $24.25 \pm 14.61$ & $\begin{array}{l}U=-4.84 \\
p<0.0001 *\end{array}$ \\
\hline MESUPES hand L (total 18) $(n=8)$ & $17.55 \pm 0.80$ & $5.75 \pm 6.67$ & $\begin{array}{l}U=-3.73 \\
p=0.0002\end{array}$ \\
\hline MESUPES total L (total 58$)(n=8)$ & $57.55 \pm 0.80$ & $30.00 \pm 20.55$ & $\begin{array}{l}U=-3.73 \\
p=0.0002 *\end{array}$ \\
\hline $\begin{array}{l}\text { Exteroception index, thumb, palm (total } \\
\text { 6) }\end{array}$ & $6.00 \pm 0.00$ & $4.13 \pm 2.30$ & $\begin{array}{l}U=-3.45, \\
p=0.0006 *\end{array}$ \\
\hline
\end{tabular}




\begin{tabular}{|lccc|} 
Proprioception wrist, index (total 6) & $8.00 \pm 0.00$ & $7.38 \pm 1.41$ & $\begin{array}{l}U=-2.33, \\
\boldsymbol{p}=0.02^{*}\end{array}$ \\
\hline $\begin{array}{l}\text { Two-point discrimination (2-3mm is a } \\
\text { normal score) }\end{array}$ & $2 \pm 1$ & $4 \pm 4$ & $\begin{array}{l}U=2.34, \\
\boldsymbol{p}=0.02^{*}\end{array}$ \\
\hline Stereognosis (total 6 objects) & $6 \pm 0$ & $3 \pm 2$ & $\begin{array}{l}U=-4.84, \\
\boldsymbol{p}<0.0001^{*}\end{array}$ \\
\hline
\end{tabular}

Legend: MMSE-short version = Mini-Mental State Examination-short version MMSE®-2-BV ${ }^{\mathrm{TM}}$ (cut-off $<13 / 16) ; \mathrm{NIHSS}=$ National Institutes of Health Stroke Scale; ${ }^{*}=p$-value $<0.05$

\section{Figures}

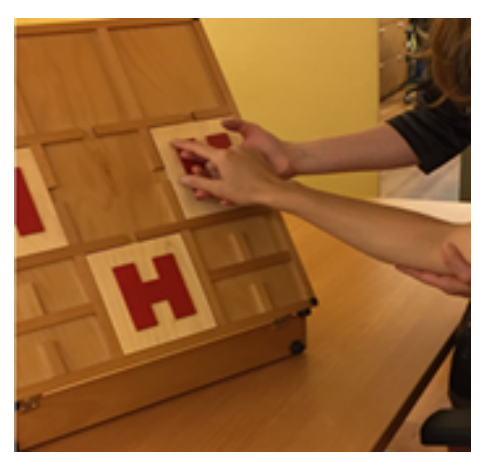

\section{Figure 1}

Example of a shape discrimination exercise The therapist guides the patient's finger along the edges to distinguish different letters " $\mathrm{H}$ ". Through reflection and attention on associated shoulder movements, the patient feels the width and length of the horizontal and vertical bars and identifies the correct letter " $\mathrm{H}$ ". This is an example of integrating attention, sensory integration, reflection and awareness of body positions, movements, and feelings. 


\section{CONSORT 2010 Flow Diagram}

\section{Enrollment}

Assessed for eligibility ( $n=26$ healthy adults; $n=14$ adults with chronic stroke)

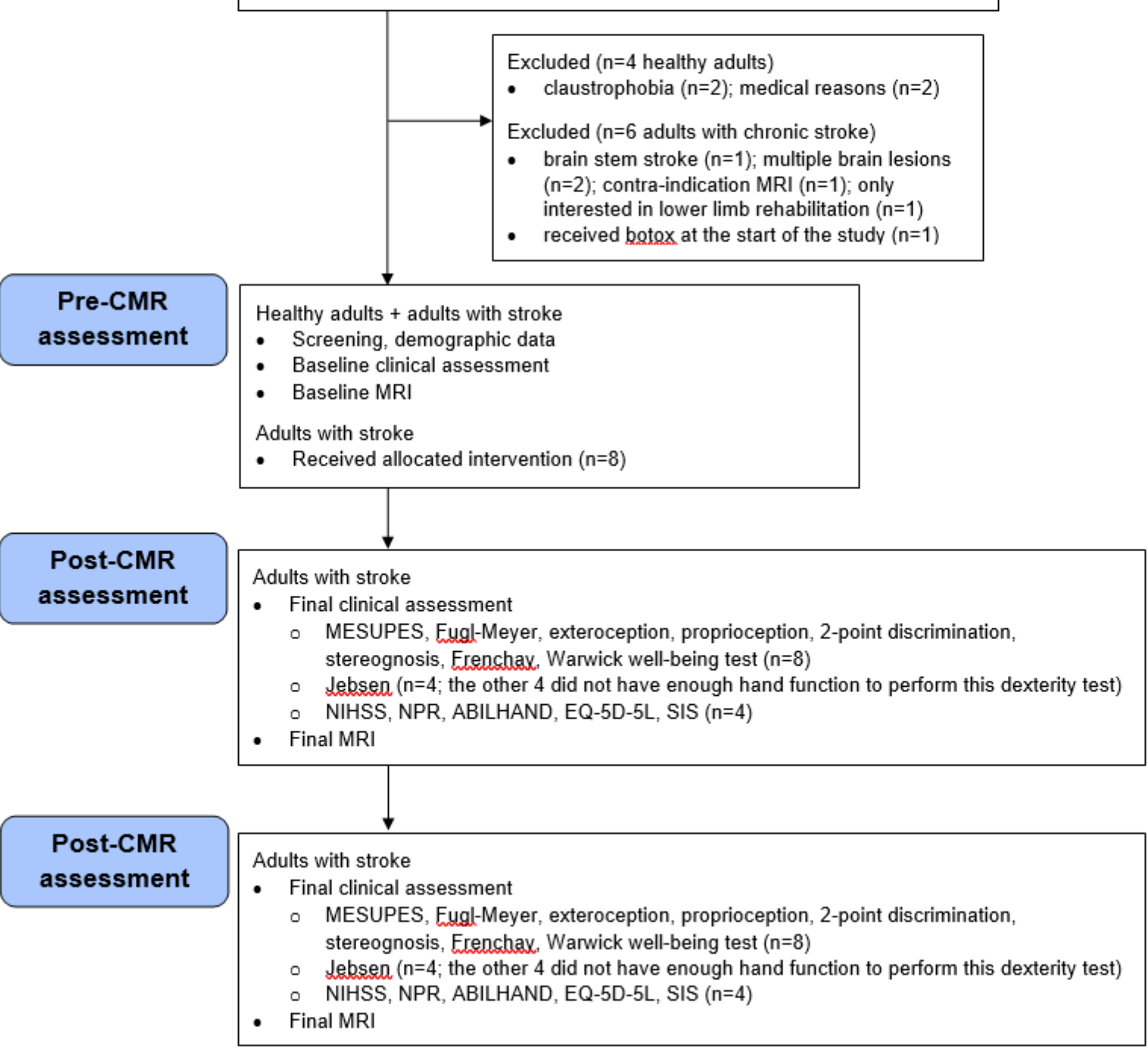

Figure 2

Flow chart Legend: MESUPES: EQ-5D-5L: Quality of life scale; Motor Evaluation Scale for Upper Extremity in Stroke Patients; MRI: Magnetic Resonance Imaging; NIHSS: National Institutes of Health Stroke Scale; NPR: Numeric Pain Rating scale; SIS: Stroke Impact Scale 
a

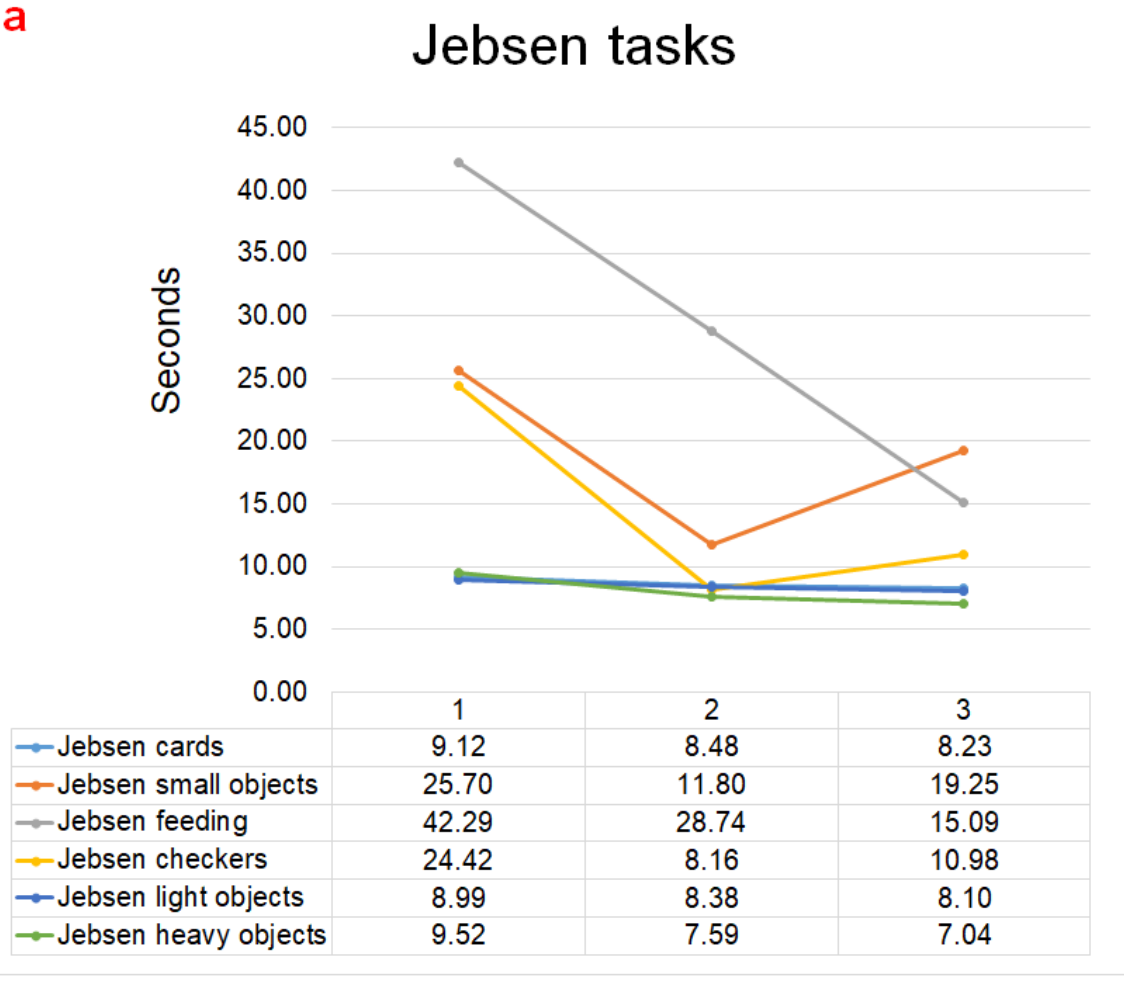

b

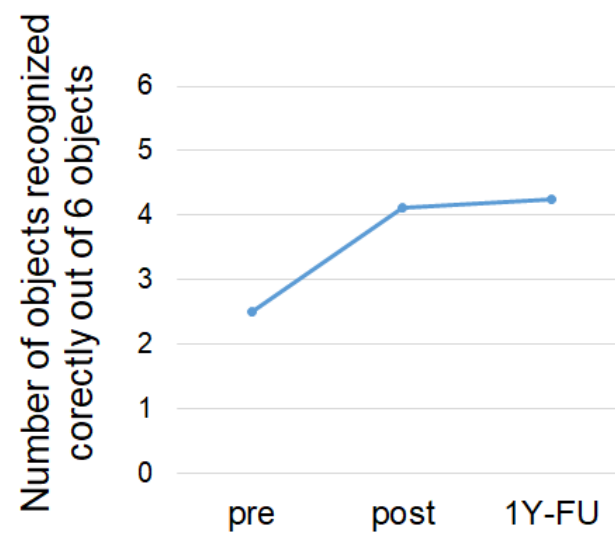

C

\section{Frenchay test}

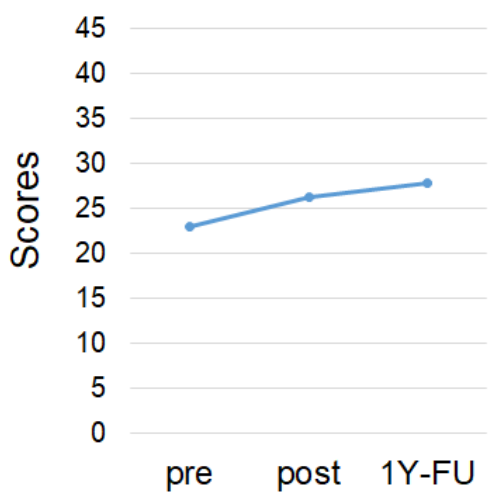

\section{Figure 3}

Behavioural outcomes of Jebsen-Taylor Hand Function Task (a), Stereognosis (b), and Frenchay test (c) (a) The Jebsen-Taylor Hand Function Task is composed of 6 activities listed in the table. The time points 1, 2 and 3 for the Jebsen tasks reflect, respectively, pre-CMR, post-CMR and at 1-year follow-up. The line graph depicts average speed for each task execution at each time point for the 4 patients with stroke who were able to perform these tasks. (b) Stereognosis was tested in all 8 patients pre-CMR, post-CMR and at 1-year follow-up. The line graph depicts the average group score of the number correctly identified objects with eyes closed out of a total of 6 objects. (c) The Frenchay test was evaluated in all 8 patients pre-CMR, post-CMR and at 1-year follow-up. The scores range from min 0 to max 45 (0-3 scoring system) and the line graph reflect the average group score at each time point. 


\section{GROC scores}

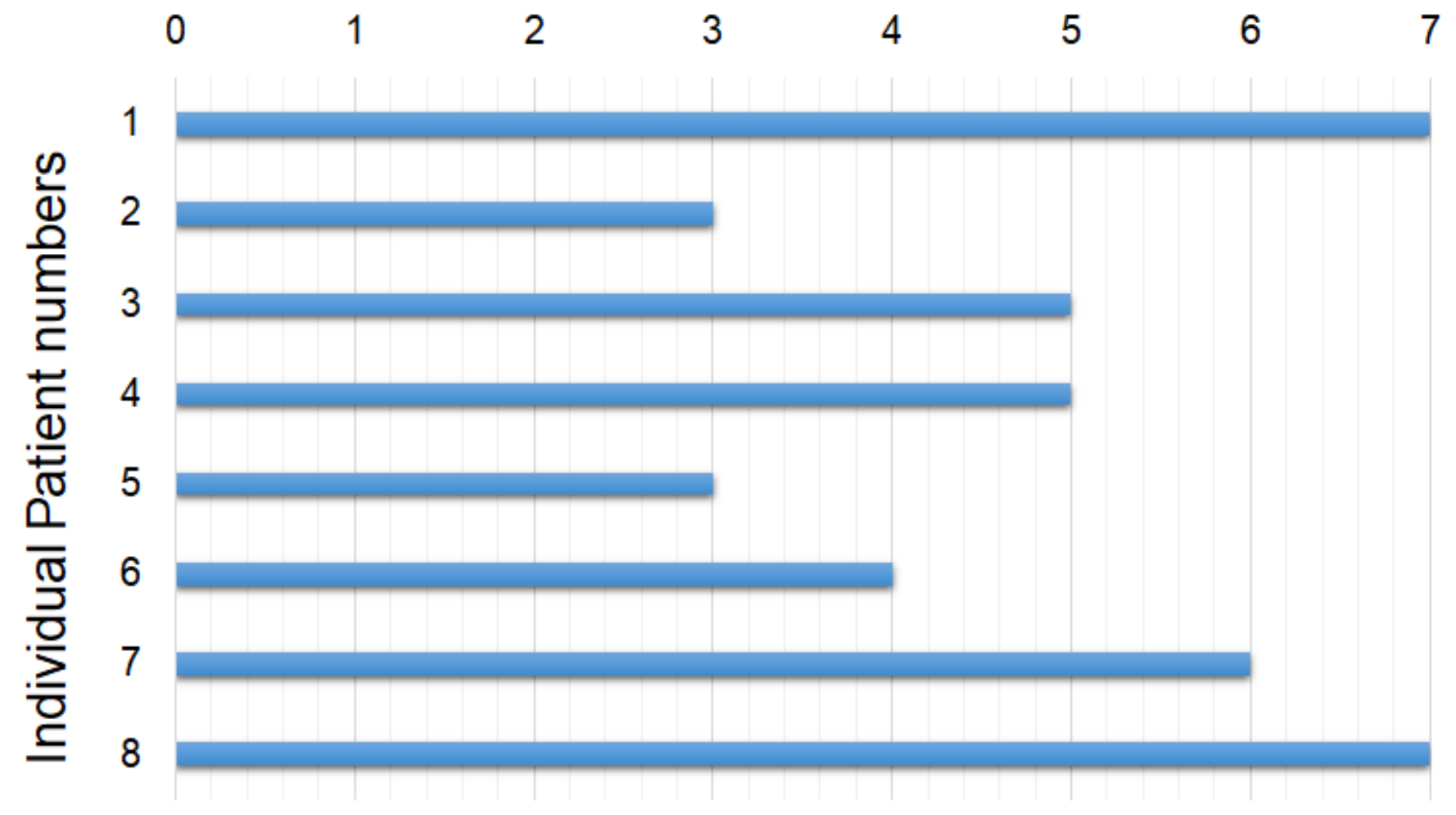

Figure 4

Global Rating of Change scale (GROC) The scale ranges from -7 (a very great deal worse) to 7 (a very great deal better). The depicted range of the 8 patients at 1 year follow-up are depicted below: score 2: a little bit better; score 3: somewhat better; score 4: moderately better; score 5: quite a bit better; score 6: a great deal better; score 7: a very great deal better. 


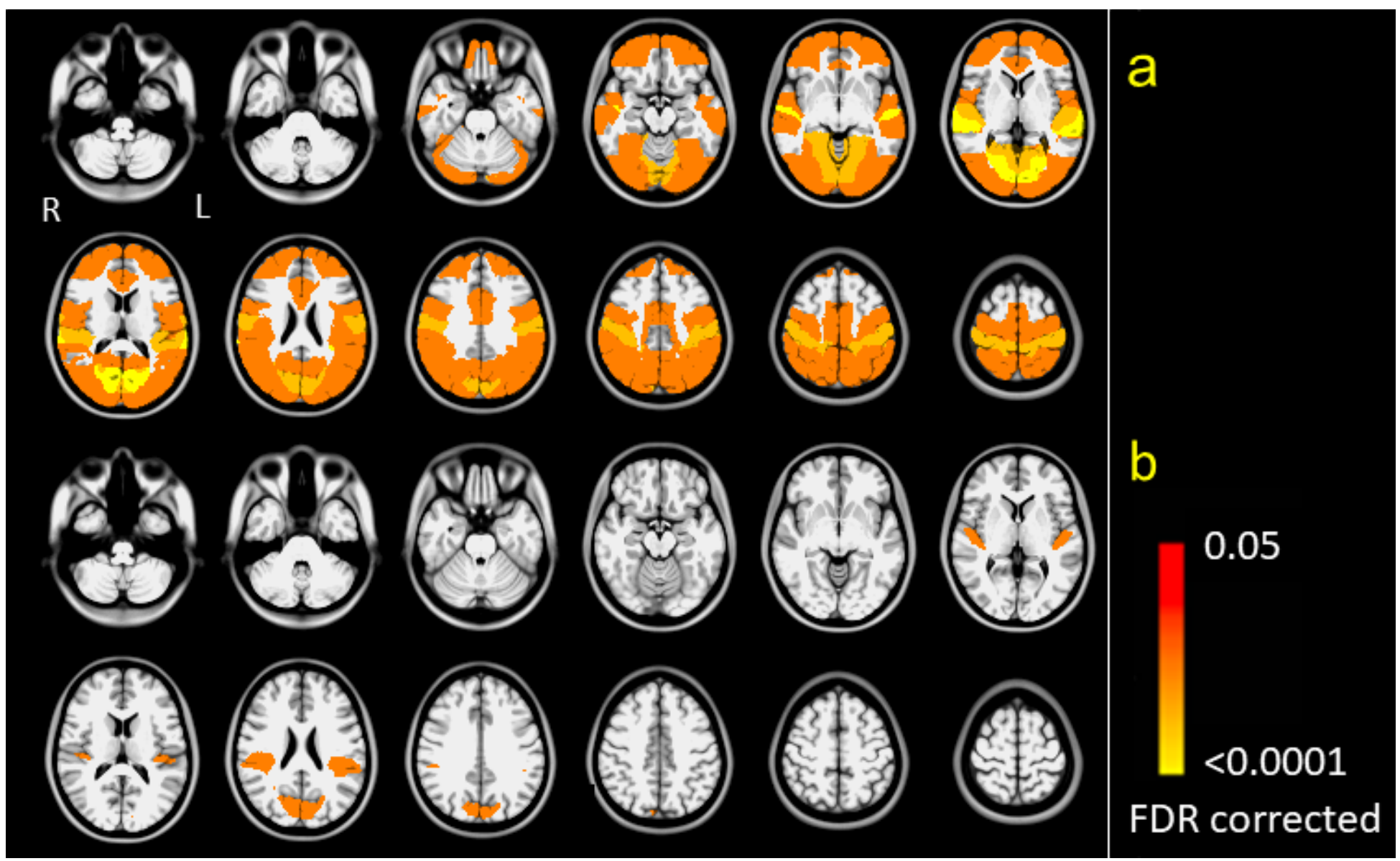

Figure 5

Connectivity before (a) and after CMR (b) (comparison stroke with healthy) Regions from the HarvardOxford cortical atlas whose functional connectivity with the OP1/OP4 seed region are statistically significantly greater (FDR-adjusted p-values $<0.05$ ) in healthy controls versus stroke pre-CMR (a) and in healthy controls versus stroke post-CMR (b). 
a Right Superior Parietal Lobe - Right Parietal Operculum

b

Right Postcentral Gyrus - Right Parietal Operculum
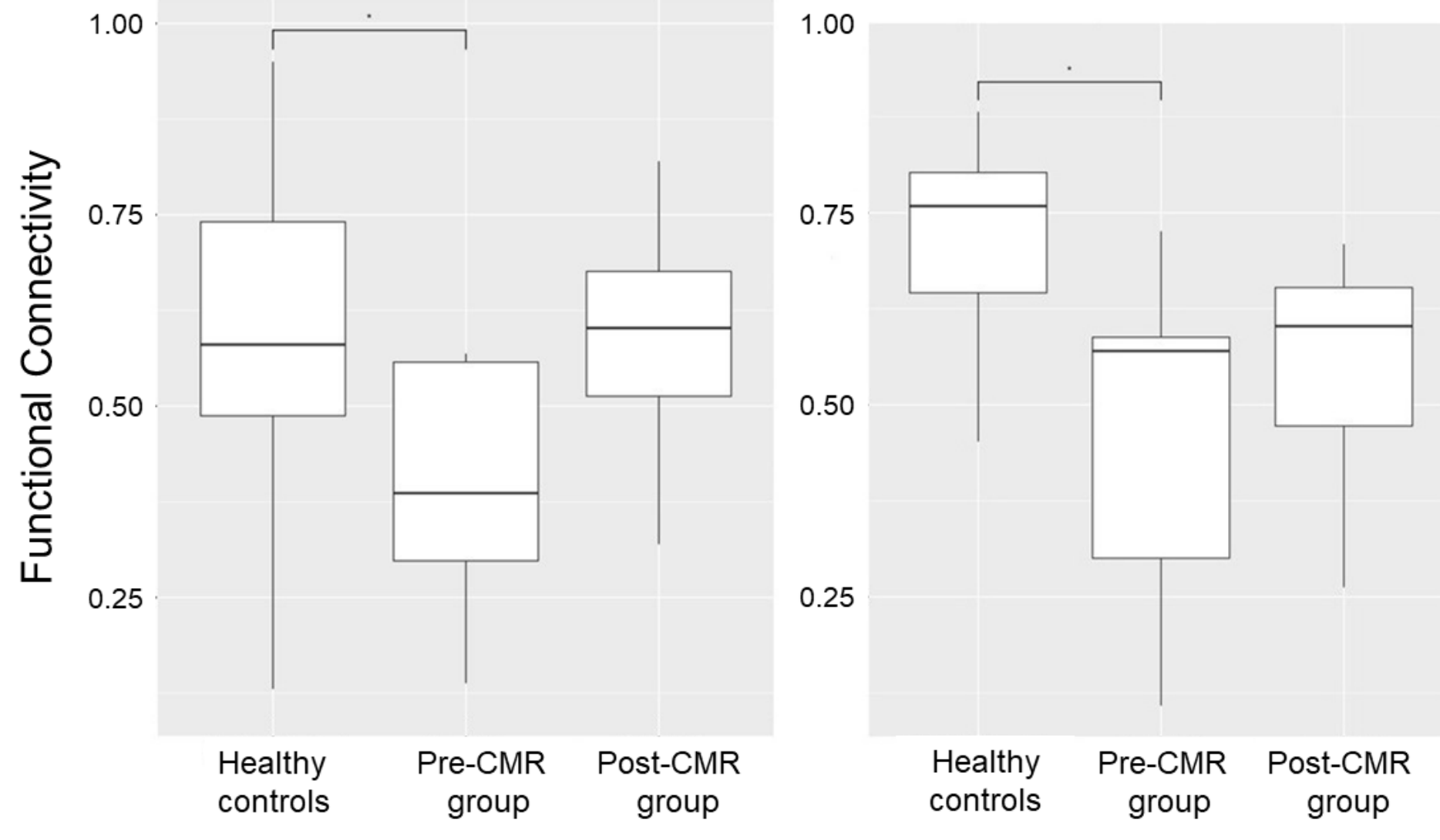

Figure 6

Boxplots of connectivity between the Right Parietal Operculum and Right Superior Parietal Lobe (a), and Right Postcentral Gyrus (b) Examples of functional connectivity for the healthy controls, pre-CMR, and post-CMR stroke groups, i.e., between the OP1/OP4 and the right postcentral gyrus (a), right superior parietal lobe (b). The star depicts a statistically significant FDR-adjusted p-values showing decreased connectivity in the stroke group at baseline (pre-CMR) compared to the healthy controls. Post-CMR, there was no statistically significant difference in connectivity between the stroke group and the healthy controls. 


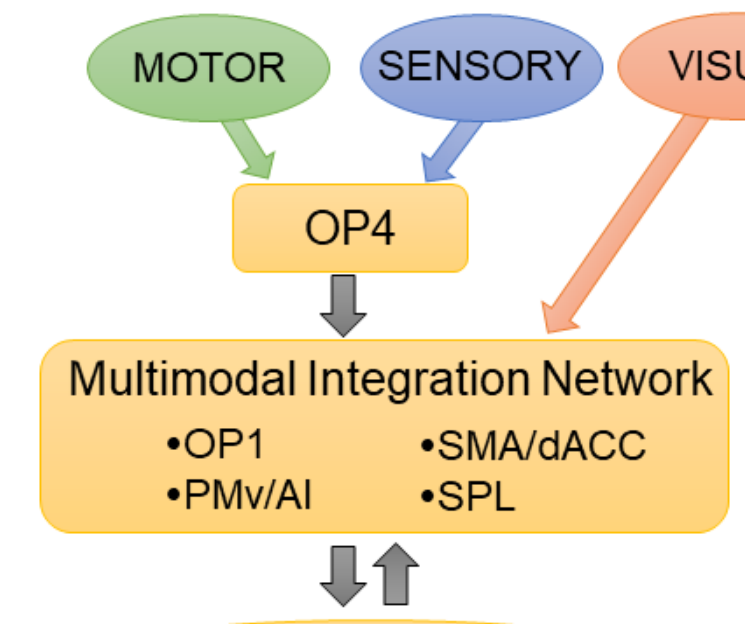

\section{Posterior Parietal Cortex (PPC)}

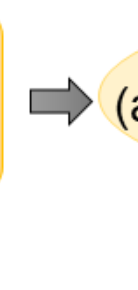

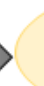

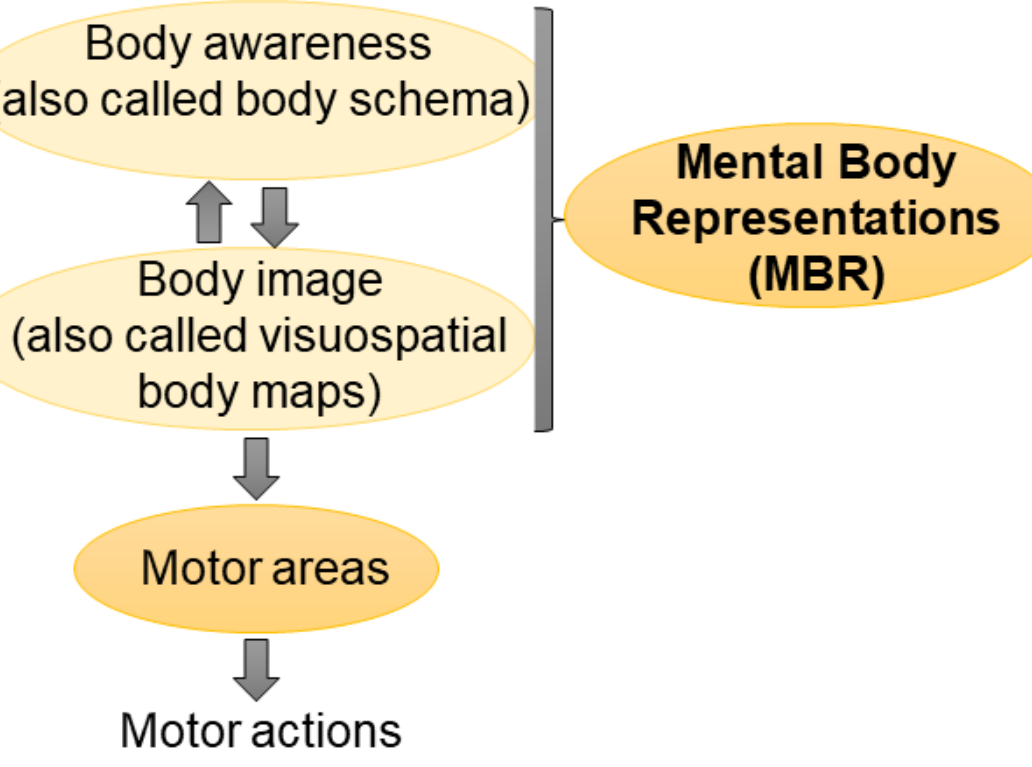

\section{Figure 7}

Proposed model to situate mental body representations within the currently known multi-sensorimotor cycle in the brain, which ultimately leads to the generation of motor actions. Legend: AG: angular gyrus; Al: anterior insula; dACC: dorsal anterior cingulate cortex; OP1/OP4: parietal operculum parts 1 and 4; PMv: ventral premotor cortex; SMA: supplementary motor area; SMG: supramarginal gyrus; SPL: superior parietal lobe.

\section{Supplementary Files}

This is a list of supplementary files associated with this preprint. Click to download.

- SupplmaterialTitlePage.docx

- SupplTableS1.docx

- SupplTables2.docx 